\title{
SPINAL CORD INJURY
}

ALBERT C. RECIO, PHILIPPINES G. CABAHUG, AND CRISTINA L. SADOWSKY

\section{CORE DEFINITIONS}

Spinal cord injury (SCI) is an insult to the spinal cord resulting in a permanent or temporary change in sensory, motor, or autonomic function. ${ }^{1-3}$ The International Standards for Neurological and Classification of Spinal Cord Injury (ISNCSCI) is a widely accepted system describing the level and extent of injury based on a systematic motor and sensory examination of neurologic function. ${ }^{1}$ The following terminology has developed around the classification of SCIs:

\section{Spinal Cord Injury Clinical Syndromes (Patterns of Incomplete Injuries)}

Central cord syndrome: Clinically presents with greater weakness of the arms than the legs, bowel, and bladder dysfunction and varying sensory loss below the injury level. It is the most common of the incomplete injury patterns often occurring in patients with underlying cervical spondylosis who sustain severe neck hypertension (most commonly from a fall). ${ }^{1,4}$

Anterior cord syndrome: Occurs as a result of interruption of the blood supply to the anterior two-thirds of the spinal cord. The clinical symptoms include a loss of motor function, pain, and temperature sensation at and below the injury level with preservation of light touch and position sense transmitted through the dorsal columns. ${ }^{1,4}$

Brown-Sequard syndrome: Results from an asymmetric cord lesion and is historically related to a knife wound injury mechanism; it is classically thought of as a hemisection of the cord although pure hemisection is uncommon with traumatic injuries. The syndrome presents with ipsilateral loss of proprioception, vibration, and motor function with contralateral loss of pain and temperature sensation. ${ }^{1,4}$

Posterior cord syndrome: Presents clinically with bilateral deficits in proprioception and vibration. Two pathological mechanisms are vitamin B12 deficiency (subacute combined degeneration) and syphilis (tabes dorsalis). ${ }^{1,4}$

Cauda equina syndrome: Involves injury to the lumbosacral nerve roots (the cauda equina or horses' tail) below spinal level lumbar L1. Injury to the nerve roots will present clinically as flaccid paralysis of the muscles in the lower limbs and areflexic bowel and bladder. All sensory modalities are similarly impaired. Sacral reflexes such as bulbocavernosus and anal wink will be absent. ${ }^{1,4,5}$

The full list of resources and references appears in the digital product found on http://connect .springerpub.com/content/book/978-0-8261-6226-7/part/part01/chapter/ch12 
Conus medullaris syndrome: May be clinically similar to cauda equina syndrome, but the injury is more rostral, involving the distal tip of the cord at the inferior aspect of L1 to L2 area. Injury to the lumbar nerve roots and conus usually results in areflexic bladder and bowel and lower limbs weakness. If it is a high conus lesion, bulbocavernosus reflex and micturition reflexes are preserved. ${ }^{1,4}$

\section{ETIOLOGY AND PATHOPHYSIOLOGY}

In the United States, most epidemiologic and demographic data have been collected by the Model Spinal Cord Injury Care Systems and are published by the National Spinal Cord Injury Statistical Center. ${ }^{6,7}$ The causes of traumatic SCI are:

- Motor vehicle crashes are the leading cause of SCI in the United States, about 37\% of cases in the database.

- Falls, 29\%, are the leading cause of SCI among the elderly.

- Violence as a cause for SCI peaked in the 1990s but has since declined to $14 \%$ (most are gunshot wounds).

- Sports injuries are reported to be $9 \%$ (most commonly: diving).

- Other causes are reported to be $11 \%$.

Spinal cord injuries usually occur in association with injuries to the vertebral column. These can include any one or more of the following: fracture of one or more of the bony elements, dislocation at one or more joints, tearing of ligaments, and disruption and/or herniation of the intervertebral disc. ${ }^{8,9}$

\section{Primary Injury}

The initial tissue disruption and damage to the spinal cord that occurs at the time of impact is the primary injury. ${ }^{8,9}$ This refers to the immediate effect of trauma, which may include:

Flexion/hyperflexion injury: Odontoid fracture, tear drop fracture, simple wedge fraction, anterior subluxation, bilateral locked facets, anterior disc space narrowing, widened interspinous distance, clay-shoveler's fracture.

Hyperextension injury: Hangman's fracture, subluxation (anterior/posterior), neural arch fracture of $\mathrm{C} 1$, anteriorly widened disc space, prevertebral swelling.

Flexion-rotation injury: Unilateral locked facets.

Vertical compression: Jefferson fracture, burst fracture.

Lateral flexion/shearing: Lateral vertebral compression, uncinate fracture, isolated pillar fracture, transverse process fracture.

Distraction: Transverse fracture through vertebra (chance fracture; most commonly seen in patients wearing lap seat belts).

\section{Secondary Injury}

Damage occurs due to pathophysiologic processes that follow SCI., ${ }^{9} 10$

Possible mechanisms of secondary injury include ischemia, edema, microvascular perfusion alterations, free radical generation, lipid peroxidation, excitotoxicity (massive glutamate release), calcium overload, inflammatory and immunologic response, and cell death (both necrotic and apoptotic-programmed cell death). 


\section{DIAGNOSTIC APPROACH}

\section{Clinical Diagnosis}

Traumatic SCI may not only be obvious from the history or initial examination but also must be excluded in patients with altered mental status (unconscious, confused, or inebriated) when the history regarding trauma is unknown. Physical examination may reveal local and radicular pain, step-off or spinal tenderness, weakness in a radicular or myelopathic distribution, sensory loss, absent tone and reflexes, and urinary retention. The presence and extent of injuries should be confirmed with spinal neuroimaging.

Documenting impairments in SCI patients is best determined by performing a standardized neurological examination as developed by the ISNCSCI. The international standards examination used for neurological classification has two components (sensory and motor). The American Spinal Cord Injury Association (ASIA) Impairment Scale (AIS) designation is used in grading the degree of impairment (Table 12.1). ${ }^{1,4}$

\section{Imaging Diagnosis}

Radiographic evaluation following spinal trauma is essential for diagnostic assessment in order to determine further care and plan for surgical decision-making. Plain x-rays of the spine may reveal fractures or dislocation. Although plain x-rays do not image the spinal cord itself, they are used to describe bony structures and alignment. Multi-slice CT is supplanting plain $\mathrm{x}$-rays in spinal trauma, due to its speed, convenience, and markedly superior sensitivity to fracture detection. MRI is the imaging modality of choice for spinal cord imaging. Myelography improves spinal cord resolution when coupled with CT. CT-angiography (CTA) demonstrates the vertebrate and carotid arteries simultaneously with spinal CT if IV contrast bolus is given. Ultrasound has been helpful intraoperatively to assess the parenchyma. ${ }^{11}$

\section{TREATMENT}

\section{Medical}

The basic principles for early hospital management of traumatic SCI include the ABCs (airway, breathing, and circulation) and spinal protection. In addition, diagnostic assessments for clinical care; assessment for associated injuries, pain, nutritional support; and management of complications such as respiratory, cardiac, genitourinary, gastrointestinal (GI), venous thromboembolism, and skin are essential. There are published guidelines recommending management following acute SCI which are included in the Consortium for Spinal Cord Medicine clinical practice guidelines on Early Management in Adults with SCI. ${ }^{12,13}$ Prevention and treatment of hypotension as soon as possible is recommended with efforts to maintain mean arterial blood pressure (MAP) greater than or equal to $85 \mathrm{mmHg}$. ${ }^{12,13}$ The guidelines state that no clinical evidence exists to definitively recommend the use of any neuroprotective pharmacologic agent including steroids in the treatment of acute SCI for functional recovery. ${ }^{14,15}$ The use of IV methylprednisolone (MP) in acute non-penetrating traumatic SCI was supported by the National Acute Spinal Cord Injury Studies (NASCIS). ${ }^{16}$ However, the results of the NASCIS trials have been challenged. The administration of MP for the treatment of SCI is not recommended..$^{17}$ 
TABLE 12.1 THE AMERICAN SPINAL CORD INJURY ASSOCIATION IMPAIRMENT SCALE

\begin{tabular}{|c|c|c|}
\hline LEVEL & NAME & DESCRIPTION \\
\hline A & COMPLETE & $\begin{array}{l}\text { No sensory or motor function is preserved in the sacral segments } \\
\text { S4-S5 }\end{array}$ \\
\hline B & $\begin{array}{l}\text { SENSORY } \\
\text { INCOMPLETE }\end{array}$ & $\begin{array}{l}\text { Sensory but not motor function is preserved at the sacral segments } \\
\text { S4-S5, and no motor function is preserved more than three levels } \\
\text { below the motor level on either side of the body }\end{array}$ \\
\hline C & $\begin{array}{l}\text { MOTOR } \\
\text { INCOMPLETE }\end{array}$ & $\begin{array}{l}\text { Motor function is preserved at the most caudal sacral segments } \\
\text { on voluntary anal contraction or the patients meet the criteria for } \\
\text { sensory incomplete status with sparing of motor function more } \\
\text { than three levels below the motor level on either side of the body. } \\
\text { This includes key or non-key muscle functions. For AIS C, less than } \\
\text { half of key muscle functions below the single NLI have a muscle } \\
\text { grade } \leq 3 \text {. }\end{array}$ \\
\hline $\mathrm{D}$ & $\begin{array}{l}\text { MOTOR } \\
\text { INCOMPLETE }\end{array}$ & $\begin{array}{l}\text { Motor function is preserved below the neurologic level, and most } \\
\text { key muscles below the NL1 have a muscle grade that is greater } \\
\text { than or equal to } 3 \text {. }\end{array}$ \\
\hline E & NORMAL & $\begin{array}{l}\text { Sensory and motor functions as tested with the ISNCSCl are } \\
\text { graded as normal in all segments, and if the patient has prior } \\
\text { deficits, then the AIS grade is } \mathrm{E} \text {. Someone without an } \mathrm{SCl} \text { does not } \\
\text { receive an AIS grade. }\end{array}$ \\
\hline
\end{tabular}

AIS, Association Impairment Scale; ISNCSCI, International Standards for Neurological and Functional Classification of Spinal Cord Injury; NLI, neurological level of injury; SCI, spinal cord injury.

\section{Surgical}

Surgical intervention involves realigning the spinal structural elements, stabilizing the spine, and decompressing compromised neural structures. The optimal timing for surgery after SCI remains a controversial topic although there is emerging evidence to suggest that early surgical decompression is safe and possibly associated with better outcomes; the Surgical Timing in Acute SCI Study (STASCIS) for cervical SCI concluded that decompression prior to 24 hours after SCI can be performed safely and is associated with improved neurological outcomes. ${ }^{18}$

\section{Rehabilitation}

An interdisciplinary team approach is important for the optimal care. Transfer to an SCI unit should be made as soon as the patient's spine is stabilized. Skilled physical and occupational therapies are geared to help the patient achieve his or her optimal level of self-care and mobility. An integrated program encompassing range of motion (ROM), strengthening, mat activities, and activities of daily living (ADL) functional training is recommended. In recent years, there has been great excitement for activity-based restorative therapy (ABRT) to achieve activity-dependent neural plasticity driven by repetitive activation of spinal cord 
pathways located both above and below the level of injury. ABRT is intended to optimize neuromuscular function and offset the rapid aging, physical deterioration, and secondary complications associated with neurological deficits. The five key components of ABRT are weight-bearing activities, functional electrical stimulation (FES), task-specific practice, massed practice, and locomotor training which includes body weight-supported treadmill walking and water treadmill training. ${ }^{19}$ Weight-bearing activities include a wide variety of activities, including tall kneeling, weight-bearing on arms while seated (sides, front, and back), quadruped (all four limbs), standing using frames or other supportive devices, and a variety of locomotor activities. FES includes upper- and lower-limb cycling and resistive exercise training via electrical stimulation of individual muscle groups. FES can also be utilized to assist movements during gait training, standing, transferring, and various other activities. Task-specific practice is key to learning a specific skill that begins with repetition of the same movement multiple times in order to develop a motor pattern. The fifth vital component of ABRT is locomotor training which includes body weight-supportive treadmill training (BWSTT) during which the participant is secured from an overhead support via an upper-body harness and trained on a moving treadmill. ${ }^{19}$

\section{Investigational Strategies}

A number of investigational strategies ${ }^{20}$ are being studied as potential treatments, and these include autologous macrophages, granulocyte colony-stimulating factor, neuroprotective agents (basic fibroblast growth factor, minocycline, riluzole), neuronal growth factors, thyrotropin-releasing hormone, and spinal cord cooling.

\section{ASSOCIATED IMPAIRMENTS AND COMPLICATIONS}

Patients with SCI are at risk for a number of medical complications which may include:

\section{Respiratory}

Pulmonary complications may include ventilatory failure, pulmonary edema, atelectasis and mucus plugs, sleep-disordered breathing (most common is obstructive sleep apnea), pulmonary embolism, and pneumonia. Pulmonary complications contribute substantively to early morbidity and mortality. Pneumonia is the leading cause of death among SCI patients. ${ }^{21}$

\section{Autonomic Nervous System}

Issues may include bradycardia, orthostatic hypotension, spinal shock, thermal dysregulation, and autonomic dysreflexia (AD). AD is a condition that is unique to SCI patients. It can occur in about $48 \%$ to $90 \%$ of SCI patients with neurologic injury at T6 or above. It is secondary to the loss of descending central sympathetic control and hypersensitivity of baroreceptors below the level of lesion. ${ }^{22}$ It's a constellation of symptoms that may include headaches, sweating, facial flushing, piloerection, irritability, or crying (in the pediatric population) associated with blood pressure elevation of 20 to $40 \mathrm{mmHg}$ higher from the baseline $(15-20 \mathrm{mmHg}$ for adolescents and children) and bradycardia. It is due to a noxious stimulus below the level of the lesion such as bladder overdistention or infection (most common), bowel impaction, 
abdominal emergencies (appendicitis, cholecystitis, pancreatitis), pressure injuries, in-grown toenails, fracture, and infections.

\section{Cardiovascular}

Cardiovascular consequences may include ischemic heart disease, peripheral vascular disease, thromboembolism, and deep venous thrombosis.

\section{Genitourinary}

Complications include urinary tract infections (UTIs), neurogenic bladder, hydronephrosis, vesicoureteral reflux, urinary stones (commonly magnesium ammonium phosphate or struvite), and pyelonephritis. UTI is the leading cause of rehospitalizations among SCI patients.

\section{Gastrointestinal}

Complications include neurogenic bowel, gastric atony, ileus, chronic constipation, fecal impaction, colonic distention, gastroesophageal reflux, cholecystitis, pancreatitis, and superior mesenteric artery (SMA) syndrome. SMA syndrome is a condition in which the third portion of the duodenum is intermittently compressed by overlying SMA resulting in GI obstruction. Gastric atony and ileus are the most common GI complication during the acute phase. Cholecystitis is the most common cause of emergency abdominal surgery in SCI patients.

\section{Metabolic and Endocrine Issues}

Glucose intolerance, hyperglycemia, hypogonadism, altered calcium metabolism (i.e., hypercalciuria, hypercalcemia), osteoporosis, altered energy consumption, and body composition are all associated with SCI. Bone health is discussed in detail in another chapter.

\section{Musculoskeletal}

Spasticity, shoulder impingement syndromes, overuse, degenerative joint disease (DJD), rotator cuff inflammation, carpal tunnel syndrome (CTS), epicondylitis, tendinitis, bursitis, Charcot spine, myofascial pain, and heterotopic ossification. The clinical practice guideline on the preservation of upper limb function provides great information. ${ }^{23}$

\section{Pain}

The International Spinal Cord Injury Pain (ISCIP) classification organizes SCI pain into three tiers: Tier 1 categorizes pain as follows: nociceptive, neuropathic, other, and unknown. Tier 2 includes various subtypes for nociceptive (visceral and musculoskeletal), and neuropathic (at level and below level). Tier 3 is used to specify the primary source and/or pathology (e.g., bursitis, CTS). ${ }^{24}$

\section{Skin}

According to the National Pressure Ulcer Advisory Panel (NPUAP), approximately 25\% of acute SCI patients will develop a pressure injury, with up to $80 \%$ of patients developing one at some point during their lifetime. Local soft tissue damage results when prolonged 
pressure over bony prominences exceeding supracapillary pressure $(70 \mathrm{mmHg})$ continuously for 2 hours. Pressure injuries are classified according to the extent of tissue damage with new revisions as of 2016. Long-standing ulcers of 20 years or longer in duration may develop squamous cell carcinoma (Marjolins ulcers).

\section{FUNCTIONAL PROGNOSIS AND OUTCOMES}

When predicting outcomes after an SCI, motor level is superior to the neurologic or sensory level in predicting function. Using the data from the U.S. Model Spinal Cord Injury System, about $13 \%$ of patients with AIS grade A admitted within 1 week post-SCI converted to incomplete status by 1 year. ${ }^{25,26}$ In patients with complete tetraplegia, more than $95 \%$ of key muscles with grade 1 or 2 at 1 -month post-SCI will reach grade 3 at 1 year. ${ }^{26}$ About $25 \%$ of the most cephalad grade 0 muscles at 1 month recover to grade 3 at 1 year. ${ }^{26}$ In patients who have complete paraplegia 1 week post-SCI, 73\% are found to have neurologic level of injury that remains unchanged at 1 year, $18 \%$ improve by one level, and $9 \%$ improve more than two levels. ${ }^{26}$ In a study done by Waters, only $5 \%$ with complete paraplegia achieve community ambulation. ${ }^{27}$

In comparison to complete injuries, the recovery is often more substantial following incomplete injuries; greater than $90 \%$ of incomplete injuries gained at least one motor level in the upper extremities compared to $70 \%$ to $85 \%$ of complete injuries. Waters reported that $46 \%$ of individuals with incomplete tetraplegia recover sufficient motor strength to be able to ambulate at 1 year post-SCI. ${ }^{27}$ Crozier reported that partial preservation of pinprick sensation below the zone of injury was predictive of eventual functional ambulation. ${ }^{28}$ The neurologic evaluation done at 72 versus 24 hours post-injury is better at predicting recovery according to a study done by Maynard et al. ${ }^{29}$

\section{RESOURCES AND REFERENCES}

The full list of resources and references appear in the digital product found on http://connect .springerpub.com/content/book/978-0-8261-6226-7/part/part01/chapter/ch12 